Research Paper

\title{
LncRNA SNHG 14 promotes cell proliferation and invasion in colorectal cancer through modulating miR-519b-3p/DDX5 axis
}

\author{
Xiaoyuan Wang1,2, Peng Yang², Dongsheng Zhang², Ming Lu¹, Chi Zhang1, Yueming Sun ${ }^{2}$ \\ 1. Department of General Surgery, The Second Affiliated Hospital Of Nanjing Medical University, Nanjing, Jiangsu Province, China. \\ 2. Department of General Surgery, The First Affiliated Hospital Of Nanjing Medical University, Nanjing, Jiangsu Province, China. \\ $\triangle$ Corresponding author: Yueming Sun, Department of General Surgery, The First Affiliated Hospital of Nanjing Medical University, Nanjing, Jiangsu Province, China. \\ E-mail: sunyueming@njmu.edu.cn. \\ (c) The author(s). This is an open access article distributed under the terms of the Creative Commons Attribution License (https://creativecommons.org/licenses/by/4.0/). \\ See http:/ /ivyspring.com/terms for full terms and conditions.
}

Received: 2020.11.05; Accepted: 2021.05.29; Published: 2021.06.11

\begin{abstract}
Numbers of studies suggest that long non-coding RNAs (IncRNAs) exert an important role in cancer progression. It is reported that IncRNA SNHGI4 (SNHG14) promotes cell proliferation and invasion in many cancers. However, the underlying molecular mechanism of SNHG14 in colorectal cancer (CRC) remains unclear. In our study, we found that SNHG14 is highly expressed in CRC tissues and cells, especially in SW480 and HT-29 cells. In addition, sh-SNHG 14 inhibits cell proliferation, cell migration and invasion, promotes cell apoptosis in CRC cell lines. Furthermore, we found that SNHG 14 functions as a sponge for miR-519b-3p, while the DEAD box protein 5 (DDX5) is a downstream target gene of miR-519b-3p, and the functions of miR-519b-3p inhibitors on the CRC progression could be rescued by downregulation of DDX5. Our findings suggest that SNHG14 promotes the CRC progression by miR-519b-3p/DDX5 axis, implying the promising therapeutic target of SNHG4 for CRC patients.
\end{abstract}

Key words: lncRNA SNHG14; miR-519b-3p; DDX5; colorectal cancer

\section{Introduction}

Colorectal cancer (CRC) causes nearly 700,000 deaths each year, making it the world's fourth killer cancer (after lung, liver and stomach) [1]. In recent years, the treatment methods of CRC have made great progress, but the mortality rate of CRC patients is still high. Therefore, it has become a research hotspot to explore the potential molecular mechanism of CRC occurrence and development, and search for new molecular therapeutic targets for CRC.

Long non-coding RNAs (lncRNAs) are a type of RNAs whose transcripts are more than 200 nucleotides in length, and have no/little potentials in coding proteins $[2,3]$. It is reported that lncRNAs play extensive regulatory roles in life activities and the occurrence and development of diseases (especially cancer, neurological diseases) [4]. Numbers of studies demonstrated that lncRNAs exert important roles in the regulation of cell proliferation, migration, invasion and apoptosis [5-7]. In recent years, studies have found lncRNAs act as important regulators in the development of CRC. For instance, lncRNA MALAT1 overexpression promotes endothelial cell formation and HIF-1 a protein expression, and participates in the occurrence and development of colorectal cancer by promoting the colorectal cancer cells mediated angiogenesis [8]. LncRNA CCAL regulates CRC occurrence and development via activating $W n t / \beta$-catenin signaling pathway through inhibition of activator protein $2 \alpha$ [9]. Additionally, the modulation of lncRNA FLANC expression affects the cell growth, apoptosis, migration, angiogenesis and metastasis formation ability of CRC cells [10].

Recent studies indicated that lncRNA SNHG14 promotes hepatocellular carcinoma progression by regulating miR-4673/SOCS1 [11]. LncRNA SNHG14 silencing inhibited non-small cell lung cancer progression via miR-34a/HMGB1 axis and promoted NSCLC cell cisplatin sensitivity [12]. Nevertheless, the function and molecular mechanism of lncRNA SNHG14 in CRC remains unclear. 
In our study, we aimed to explore the function and potential underlying mechanism of SNHG14 in CRC. Subsequently, the influences of SNHG14 knockdown on CRC progression. Finally, we found that SNHG14 promotes cell proliferation and invasion in colorectal cancer through modulating miR-519b-3p/DDX5 axis. Our study will provide an experimental basis for the molecular mechanism of CRC and offer therapeutic targets against the CRC.

\section{Materials and methods}

\section{Clinical samples}

A total of 30 paired CRC tumor tissues and adjacent non-tumor tissues were obtained from the Second Affiliated Hospital of Nanjing Medical University for this study. Patients older than 18 years and those who had undergone $\mathrm{R} 0$ resection were eligible for the study; however, those with metastasis, a concurrent diagnosis of familial adenomatous polyposis, lynch syndrome or irritable bowel disease (IBD), or metachronous or synchronous CRC were excluded from the analysis. All patients did not get treatment. Each patient had signed a written informed consent. The First School of Clinical Medicine, The First Affiliated Hospital of Nanjing Medical University Ethics Committees approved this work. The Ethics Committees approved protocol number was 2019-SRFA-131. In addition, detailed clinic parameters had been shown in the Table 1 .

Table 1. The detailed clinic parameters of enrolled patients

\begin{tabular}{|c|c|c|c|}
\hline \multirow{2}{*}{$\begin{array}{l}\text { Clinical } \\
\text { parameters }\end{array}$} & \multicolumn{2}{|l|}{ SNHG14 } & \multirow[t]{2}{*}{ P value } \\
\hline & Low expression $(n=14)$ & High expression $(n=16)$ & \\
\hline Age & & & 0.165 \\
\hline$>60$ & 8 & 9 & \\
\hline$\leq 60$ & 6 & 7 & \\
\hline Gender & & & 0.352 \\
\hline Male & 8 & 8 & \\
\hline Female & 6 & 8 & \\
\hline Tumor Size & & & $0.026^{*}$ \\
\hline$>2 \mathrm{~cm}$ & 4 & 7 & \\
\hline$\leq 2 \mathrm{~cm}$ & 10 & 9 & \\
\hline Tumor stage & & & $0.039^{*}$ \\
\hline $\mathrm{I} \sim \mathrm{II}$ & 9 & 4 & \\
\hline III IV & 5 & 12 & \\
\hline \multicolumn{2}{|c|}{ Lymph nodes metastasis } & & 0.068 \\
\hline No & 5 & 6 & \\
\hline Yes & 9 & 10 & \\
\hline \multicolumn{2}{|c|}{ Distant metastasis } & & $0.028^{*}$ \\
\hline No & 10 & 5 & \\
\hline Yes & 4 & 11 & \\
\hline
\end{tabular}

\section{Establishment of colorectal cancer model}

20 male NOD-SCID mice aged at 8 weeks were used to establish model in the experiment. These mice were purchased from Charles River Laboratories (Beijing, China). The transfected HT-29 cells $\left(5 \times 10^{6}\right.$ cells in $100 \mu \mathrm{L}$ PBS) were injected subcutaneously into the forelimbs of nude mice. All the mice were observed every 2 days for at least 7 weeks. All procedures were conformed to the Guide for the Care and Use of Laboratory Animal published by the US National Institutes of Health (NIH publication, 8th edition, 2011), and approved in accordance with the Committee on Animal Care and Use of ethical committee of Nanjing Medical University. Ethical number of animal experiment is IACUC-1903018. The nude mice were housed in 12-h light/dark cycle with a free access to standard chow and tap water in a temperature-controlled room.

\section{Cell culture and transfection}

Human CRC cell lines (SW480, HT-29, HCT-8 and DLD-1) and human normal colon epithelial cells (NCM460) were purchased from the American Type Culture Collection (ATCC; Manassas, VA, USA). All the cells were cultured in RPMI 1640 medium supplemented with $10 \%$ fetal bovine serum (FBS), 100 $\mathrm{U} \mathrm{mL}^{-1}$ penicillin, and $100 \mathrm{~g} / \mathrm{mL}$ streptomycin and incubated at $5 \% \mathrm{CO}_{2} ; 37^{\circ} \mathrm{C}$.

Short hairpin RNA (shRNA) targeting SNHG14 (sh-SNHG14), sh-DDX5 and the corresponding negative controls (sh-NC) were designed and synthesized by Thermo Fisher Scientific (Shanghai, China). MiR-519b-3p mimics, miR-519b-3p inhibitor and the corresponding negative controls (miR-NC) were purchased from Thermo Fisher Scientific. These plasmids were transfected into cells by using Lipofectamine 2000 (Invitrogen, CA, USA) according to the manufacturer's instruction.

\section{qRT-PCR assay}

Total RNAs of the samples were extracted by Trizol reagent (Takara, Otsu, Japan) following the manufacturer's protocols. Reverse transcriptase reactions were performed using HiScript ${ }^{\circledR}$ III 1st Strand cDNA Synthesis Kit (Vazyme, Nanjing, China). All qRT-PCR experiments were performed using SYBR green reagents (Vazyme, Nanjing, China) and a qRT-PCR Detection System (Analytic, jena, Germany). The quantitative measures were obtained using the $2^{-\Delta \Delta \mathrm{CT}}$ method and were normalized to either $\beta$-actin or U6 level. The primers for RT-qPCR were as follows: hsa-SNHG14: 5'-GGGTGTTTACGTAGACC AGAACC-3' (Forward) and 5'- CTTCCAAAAGCCTT CTGCCTTAG-3' (Reverse); hsa-miR-519b-3p: 5'-GGT CAAGTGACACCGTCG-3' (Forward) and 5'-TGCAG CTGGGGGTCAG-3' (Reverse); hsa-DDX5: 5'-GGCCT GATCACAGAACCATT-3' (Forward) and 5'-ACC ACCCTTATTCCCAAACC-3' (Reverse); hsa-U6: 5'-GTGATCACTCCCTGCCTGAG-3' (Forward) and 5'-GGACTTCACTGGACCAGACG-3' (Reverse); 
hsa-GAPDH: 5'-CCGCATCTTCTTGTGCAGTG-3' (Forward) and 5'-CCCAATACGGCCAAATCCGT-3' (Reverse).

\section{CCK-8 assay}

Cell Counting Kit8 (CCK-8; Apexbio, HOU, USA) was used to detect CRC cells growth. Briefly, SW480 and HT-29 cells $\left(5 \times 10^{3}\right.$ cells/well) were incubated in 96-well for 24,48 or $72 \mathrm{~h}$. Then, the cells were treated with certain drugs for $24 \mathrm{~h}$, and $10 \mu \mathrm{L}$ of CCK-8 solution was subsequently added to each well. Then the plate was incubated at $37^{\circ} \mathrm{C}$ for $3 \mathrm{~h}$. The optical density (OD) value was measured at $450 \mathrm{~nm}$ by ultraviolet spectrophotometer (Thermo Fisher Scientific, Inc.).

\section{EdU assay}

Cell proliferation was assessed by EdU assay (Solarbio, Beijing, China). SW480 and HT-29 cells $\left(2 \times 10^{4}\right.$ cells/well) were seeded on the glass coverslips in 24-well plates and incubated in RPMI 1640 containing $10 \%$ FBS for $24 \mathrm{~h}$. EdU was added to each well for $2 \mathrm{~h}$. After fixed, the cells were stained by $1 \times$ Apollo for 30 min in dark. Next, DAPI was used to stain nucleus for $5 \mathrm{~min}$. In the end, cells were observed under fluorescence microscope (Olympus, Tokyo, Japan).

\section{Western blot assay}

Total proteins were extracted with ice-cold RIPA lysis buffer plus PMSF. Total protein concentrations were detected with BCA assay kit (Santa Cruz, California, USA). Prepared protein samples were separated in $10 \%$ sodium dodecyl sulfate polyacrylamide gel electrophoresis (SDS-PAGE), and transferred into $0.22 \mu \mathrm{m}$ PVDF membranes. After blocked with skim milk, these membranes were incubated with primary antibodies at $4{ }^{\circ} \mathrm{C}$ overnights, followed by incubation with the appropriate secondary antibody for $1 \mathrm{~h}$ at room temperature. Finally, the enhanced chemiluminescence (ECL, Pierce, Rockford, IL) visualized this membrane. The primary antibodies were anti-PCNA, anti-Ki-67, antiBcl-2, anti-Bax, anti-cleaved caspase-3, anti-cleaved caspase-9, anti-Cox-2, anti-MMP-2, anti-MMP-9, anti-DDX5 and anti- $\beta$-actin (Abcam, Cambridge, UK).

\section{Cell apoptosis assay}

Cell apoptosis was tested by the Annexin V-FITC kit (Thermo Fisher Scientific, Inc.). First, SW480 and HT-29 cells were collected, resuspended in cold PBS, centrifugated at $1000 \mathrm{rpm}$ in room temperature for 5-10 min. Then, cells were incubated with $5 \mu \mathrm{L}$ AnnexinV-Alexa Fluor 647 for $15 \mathrm{~min}$ and then co-incubated with $5 \mu \mathrm{L}$ PI before detection. Finally, the apoptosis rate was analyzed via flow cytometer
(Beckman Coulter, CA, USA).

\section{Transwell chamber assay}

Cell invasion and migration were detected by transwell assay. The transwell membrane was covered with (invasion) or without (migration) matrigel. Transwell lower chamber was put $600 \mu \mathrm{L}$ RPMI-1640 medium containing 10\% FBS, added 200 $\mathrm{mL}$ cell suspension to the upper chamber for $24 \mathrm{~h}$. After incubation for $48 \mathrm{~h}$, invaded or migrated cells were fixed with methanol and stained with $0.1 \%$ crystal violet. Finally, the cells were observed under a microscope (Olympus, Tokyo, Japan) and counted.

\section{Luciferase reporter assay}

The wild-type reporter of the 3'UTR region of DDX5 (DDX5-WT), the wild-type SNHG14 reporter (SNHG14-WT), the mutant-type SNHG14 reporter (SNHG14-Mut) and the mutant-type DDX5 reporter (DDX5-Mut) were purchased from Synthgene Biotech (Nanjing, China). SNHG14-WT or SNHG14-Mut were co-transfected with miR-519b-3p mimic or miR-NC into SW480 and HT-29 cells with Lipofectamine 2000. DDX5-WT or DDX5-Mut were also co-transfected miR-519b-3p mimic or miR-NC into SW480 and HT-29 cells. After transfection for $48 \mathrm{~h}$, the relative luciferase activities of cells were measured by the dual Glo Luciferase Assay System (Promega, Shanghai, China) in accordance with the manufacturer's introductions. Renilla signals were used to normalize luciferase activity.

\section{HE staining}

These samples were collected from sh-NC and sh-SNHG14 group. The sample paraffin sections were dewaxed and hydrated; and then slices were incubated in hematoxylin solution for $15 \mathrm{~min}$ followed washed with PBS. Secondly, the slices were differentiated, stained by eosin solution for 15s. In the end, the slices were dehydrated, transparentized and sealed, the images were captured under an optical microscope (Olympus, Tokyo, Japan).

\section{Immunohistochemistry}

The sliced tumors were dewaxed, hydrated and incubated with $3 \% \mathrm{H}_{2} \mathrm{O}_{2}$ for 10 min in order to block endogenous peroxidase activity, and washed with PBS. The slices were incubated with primary antibody against Ki-67 (1:100) overnight at $4{ }^{\circ} \mathrm{C}$, and then incubated with secondary antibodies at room temperature for $1-2 \mathrm{~h}$, washed with $1 \times$ TBST and incubated in diaminobenzidine chromogen for $5 \mathrm{~min}$, and the nucleus counterstained with hematoxylin. The slices were observed under microscope (Olympus, Tokyo, Japan). Ki-67 positive staining was developed to produce brown reaction product. 


\section{TUNEL staining}

The sample paraffin sections were dewaxed and hydrated; the endogenous peroxidase was inactivated by hydrogen peroxide. Next, tunel test solution labeled sample biotin followed incubated with DAB for 5-30 $\mathrm{min}$, and then the slices were incubated in hematoxylin solution for $15 \mathrm{~min}$ followed washed with PBS. Finally, the slices were dehydrated transparentized, sealed and observed under optical microscope (Olympus, Tokyo, Japan).

\section{Statistical analysis}

Data was presented as the mean \pm standard deviation (SD) of at least 3 independent experiments. Student's $t$-test was performed to determine the significance between two groups, or one-way ANOVA with Bonferroni post-hoc tests for multiple groups, ${ }^{*} P<0.05$ and ${ }^{* *} P<0.01$ were considered as significant.

\section{Results}

\section{Clinical parameters of enrolled CRC patients in our study}

The clinicopathological information of enrolled 30 CRC patients was presented in Table 1. As shown in Table 1, we found that high SNHG14 expression was strongly correlated with tumor stage, tumor size and distant metastasis. However, there were no significant differences between age, gender and lymph node metastasis.

\section{SNHG 14 is highly expressed in CRC tissues and cells}

To detect the correlations between the expression levels of SNHG14 and the occurrence/development of CRC, we firstly analyzed the expression of SNHG14 in CRC tissues and cells, individually. We found that SNHG14 expression was significantly upregulated in CRC tissues compared
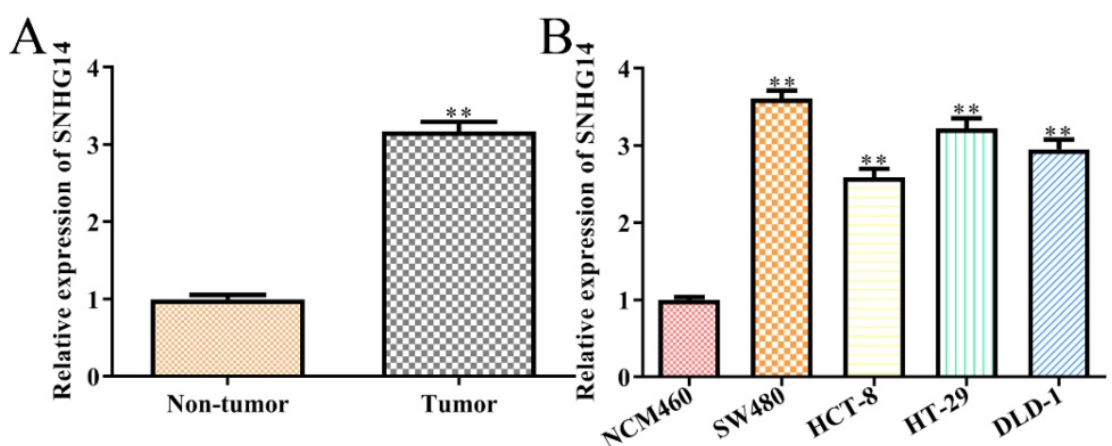

Figure 1. SNHG14 is highly expressed in CRC tissues and cells. (A) qRT-PCR showed expression of SNHG14 in tumor tissues and adjacent non-tumor tissues. $* * P<0.01$ vs. Non-tumor tissue. $n=6$. (B) $q$ RT-PCR tested the expression of SNHG 14 in CRC cell lines (SW480, HCT-8, HT-29 and DLD-1) and normal human colon epithelial cells (NCM460). Values are mean \pm SEM $* * P<0.01$ vs. NCM460. $n=3$ per group. with adjacent non-tumor tissues (Fig. 1A). As shown in Fig. 1B, the expression of SNHG14 was remarkably increased in CRC cells (SW480, HCT-8, HT-29 and DLD-1) compared to normal human colon epithelial cells (NCM460). Considering that the SNHG14 expression was dramatically higher in SW480 and HT-29 cells than other CRC cells (Fig. 1B). Therefore, SW480 and HT-29 cells were selected for subsequent experiments.

\section{Knockdown of SNHG 14 inhibits the proliferation and promotes the apoptosis of CRC cells}

To investigate the biological function of SNHG14 in CRC, sh-SNHG14 and its scramble control (sh-NC) were transfected into SW480 and HT-29 cells, respectively. The knockdown efficiency of sh-SNHG14 in SW480 and HT-29 cells were validated by qRT-PCR analysis and were presented in Fig. 2A. Functionally, CCK-8 and EdU assays showed that sh-SNHG14 significantly inhibited the proliferation of SW480 and HT-29 cells (Fig. 2B-C). Furthermore, PCNA and Ki-67, the proliferation-related proteins, were markedly downregulated by sh-SNHG14 in SW480 and HT-29 cells (Fig. 2D). Next, flow cytometry assay indicated that sh-SNHG14 promoted cell apoptosis in SW480 and HT-29 cells (Fig. 2E). In addition, the expression of apoptosis-related proteins was examined by western blot analysis. We found that sh-SNHG14 reduced the expression level of anti-apoptotic protein (Bcl-2), while elevated the expression levels of pro-apoptosis proteins (Bax, Cleaved caspase-3 and Cleaved caspase-9) in SW480 and HT-29 cells (Fig. 2F). These results indicated that sh-SNHG14 inhibits the proliferation and promotes the apoptosis of CRC cells.

\section{Knockdown of SNHG 14 inhibits migration and invasion of CRC cells}

As shown in Fig. $3 \mathrm{~A}$ and $3 \mathrm{~B}$, scratch and transwell chamber assays showed that the migration and invasion abilities of SW480 and HT29 cells were remarkably restrained in cells transfected with sh-SNHG14, when compared to scramble control. Besides, western blot analysis revealed that sh-SNHG14 downregulated the expression of migration and invasion-related proteins (Cox-2, MMP-2 and MMP-9) in SW480 and HT-29 cells (Fig. 3C). All these results illustrated that sh-SNHG14 inhibited migration and invasion of CRC cells. 

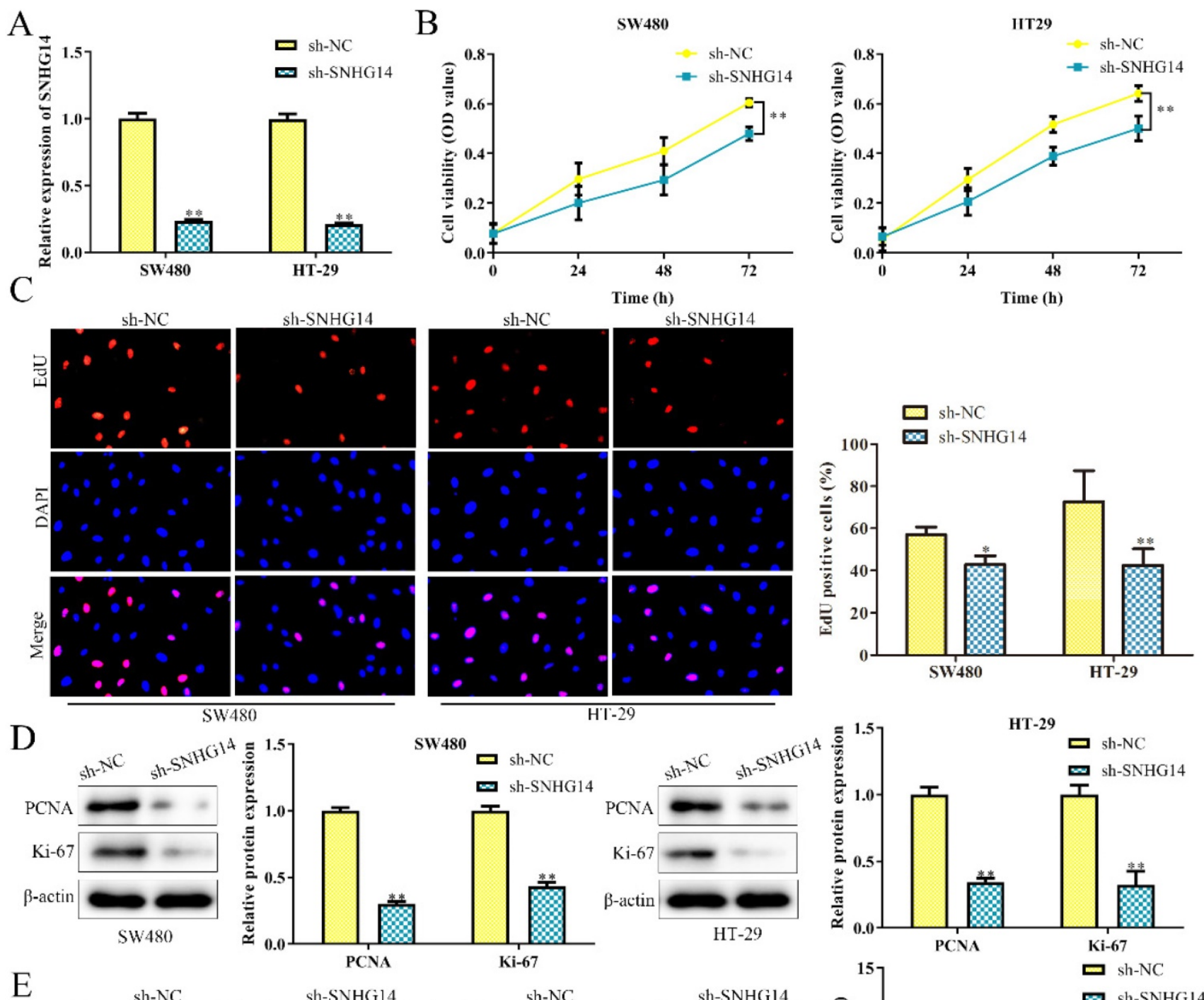

$\mathrm{E}$
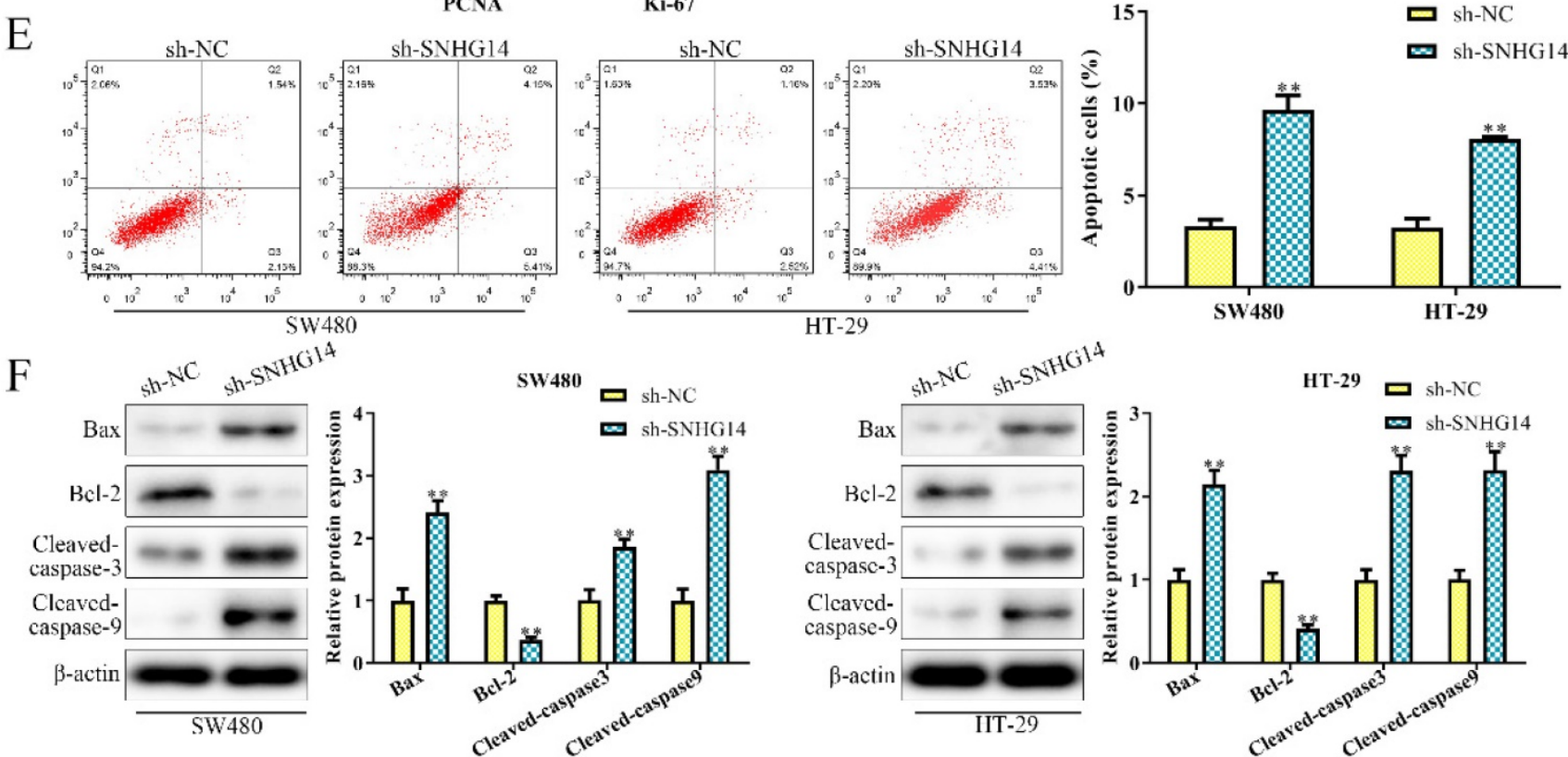

Figure 2. Sh-SNHG14 inhibits cell proliferation and promotes cell apoptosis in CRC. SW480 and HT-29 cells were transfected with sh-SNHG14. (A) qRT-PCR analysis to detect the knockdown efficiency of SNHG14. (B) CCK-8 assays for the cell proliferation at the indicated time points (24, 48 and $72 \mathrm{~h}$ ). (C) EdU assays for the cell proliferation. (D) Western blot analysis of the expression levels of proliferation-associated proteins (PCNA and Ki-67). (E) Flow cytometry analyzed the apoptosis rate. (F) Western blot analysis of the expression levels of apoptosis associated proteins (Bax, Bcl-2, Cleaved caspase-3 and Cleaved caspase-9). Values are mean $\pm \mathrm{SE}$, $* P<0.05, * * P<0.01$ vs. sh-NC, $\mathrm{n}=3$ per group. 

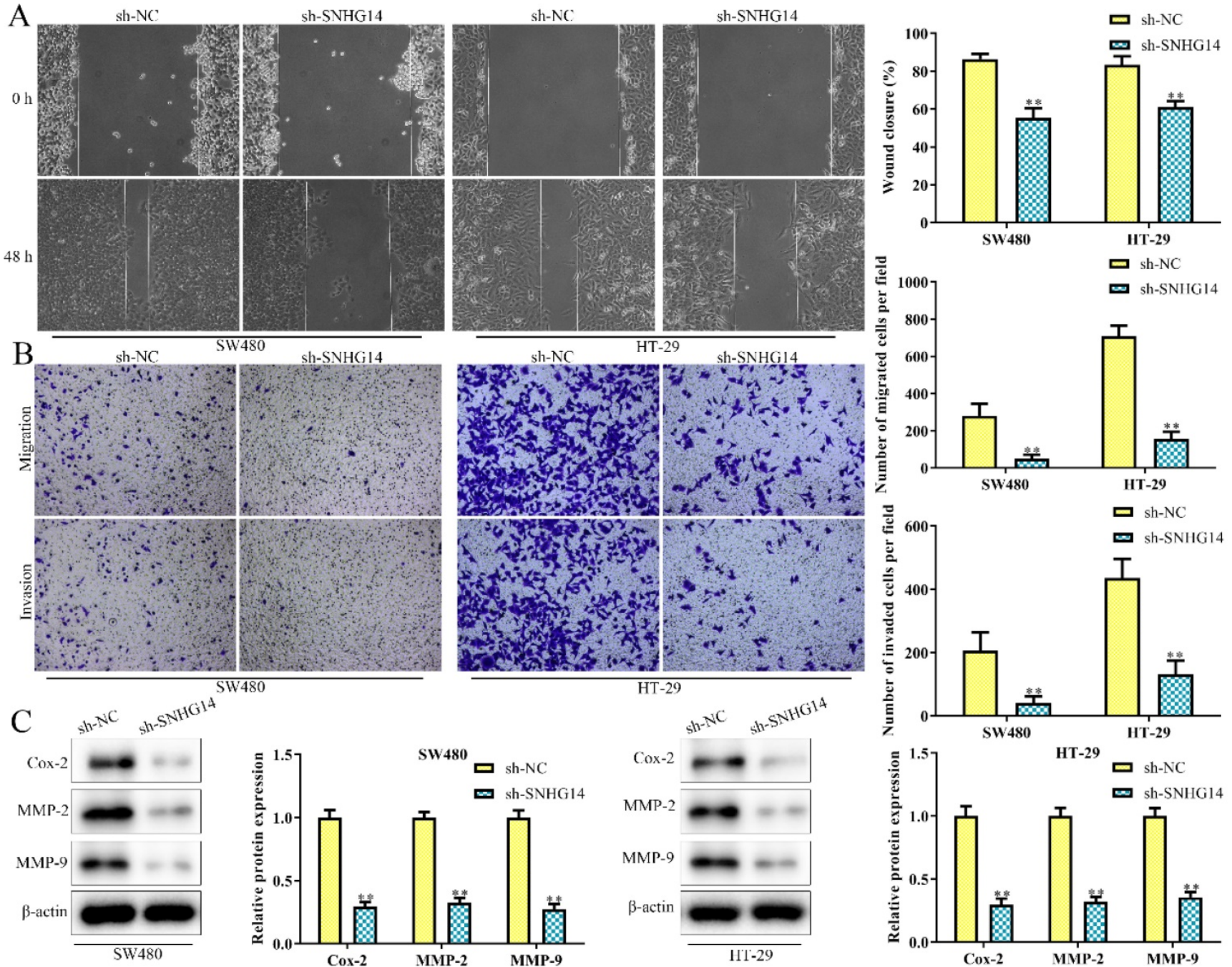

Figure 3. Sh-SNHG 14 inhibits cell migration and invasion in CRC. SW480 and HT-29 cells were transfected with sh-SNHG14. (A, B) Scratch test and Transwell chamber assay for the migration and invasion abilities. (C) Western blot analysis of the expression levels of migration- and invasion-associated proteins (Cox-2, MMP-2 and MMP-9). Values are mean $\pm \mathrm{SE}, * * P<0.01$ vs. sh-NC, $\mathrm{n}=3$ per group.

\section{SNHG 14 sponges and targets on the miR-519b-3p in CRC cells}

Then we studied the molecular mechanism of SNHG14 in CRC. Previous studies have shown that lncRNAs could serve as ceRNAs by sponging miRNAs $[13,14]$. Hence, we used StarBase analysis to predict the potential miRNA, which interacted with SNHG14, and finally selected miR-519b-3p as a potential target (Fig. 4A), since its dual functions as either oncogene or tumor suppressor in the regulation of the proliferation, migration and apoptosis of tumor cells [15]. To evaluate the interactions between miR-519b-3p and SNHG14, luciferase reporter assay was performed and revealed a significant decrease in luciferase activity of mutant SNHG14 in cells co-transfected with SNHG14-WT and miR-519b-3p mimic (Fig. 4B). However, no significant difference was observed in luciferase activity between the miR-519b-3p mimic group and control group when the putative binding sites were mutated (Fig. 4B). Consistently, sh-SNHG14 could increase miR-519b-3p expression in SW480 and HT-29 cells (Fig. 4E). Subsequently, we discovered that miR-519b-3p expression was notably upregulated in non-tumor groups compared to tumor tissues (Fig. 4C). Moreover, RT-PCR indicated that miR-519b-3p expression was remarkably decreased in CRC cells (SW480, HCT-8, HT-29 and DLD-1) compared to NCM460 cells (Fig. 4D). These findings revealed that SNHG14 functions as a sponger for the miR-519b-3p in CRC cells.

\section{MiR-519b-3p inhibits cell proliferation, migration/invasion and promotes apoptosis in CRC cells}

In order to dissect the biological function of miR519b-3p in CRC progression, miR-519b-3p mimic and NC mimic were transfected into SW480 and HT-29 cells, respectively. qRT-PCR assay was used to 
confirm the overexpression efficiency of miR-519b-3p mimic in SW480 and HT-29 cells. We found that miR-519b-3p mimic triggered an obvious upregulation of miR-519b-3p expression in SW480 and HT-29 cells, when compared to NC mimic (Fig. 5A). CCK-8 and EdU assays indicated that miR-519b-3p mimic remarkably decreased the proliferation of SW480 and HT-29 cells (Fig. 5B-C). In addition, flow cytometry assay elucidated that miR-519b-3p overexpression promoted cell apoptosis in SW480 and HT-29 cells (Fig. 5D). Transwell chamber assay further demonstrated that the migration and invasion abilities of cells were obviously repressed in SW480 and HT-29 cells transfected with miR-519b-3p mimic (Fig. 5E). All these results illustrated that miR-519b-3p overexpression inhibited the cell proliferation, migration/invasion, while promoted the cell apoptosis in CRC.

\section{DDX5 is a downstream target gene of miR-519b-3p}

To explore the regulatory mechanism through which miR-519b-3p affected CRC progression, we used TargetScan to predict miR-519b-3p target genes and identified DDX5 as a potential target (Fig. 6A), because of its proto-oncogenic role in human cancers [16-18]. To determine the interaction of miR-519b-3p and DDX5, we performed luciferase reporter analysis and found significantly reduction in the luciferase activity of 3'UTR region of DDX5 when the cells were co-transfected with DDX5-WT and miR-519b-3p mimics. However, there was no notable difference in luciferase activity of DDX5-Mut between miR-519b-3p mimics group and control group (Fig. 6B). To further study the correlation of DDX5 and miR-519b-3p, we detected that DDX5 expression was decreased by miR-519b-3p mimics in SW480 and HT-29 cells via qRT-PCR and western blot (Fig. 6C-D). In addition, we found that DDX5 expression was significantly upregulated in tumor tissues compared to non-tumor groups, and increased in CRC cells (SW480, HCT-8, HT-29 and DLD-1) compared to NCM460 cells (Fig. $6 \mathrm{E})$. These results demonstrated that DDX5 is a downstream target gene of miR-519b-3p.

\section{MiR-519b-3p/DDX5 axis mediates the SNHG 14 knockdown-induced inhibition of CRC progression}

To explore whether SNHG14 promoted the CRC progression through regulating miR-519b-3p/DDX5 pathway, we performed rescue experiments using miR-519b-3p inhibitors and sh-DDX5. qRT-PCR assay was used to confirm the inhibition efficiency of miR-519b-3p inhibitors and sh-DDX5 in SW480 and HT-29 cells transfected with sh-SNHG14. We found that miR-519b-3p and DDX5 expression was downregulated treated with miR-519b-3p inhibitors and sh-DDX5 in SW480/sh-SNHG14 and HT-29/shSNHG14 cells (Fig. 7A). CCK-8 and EdU assays demonstrated that sh-DDX5 could rescue miR-519b-3p inhibitors-induced activation of the proliferation of SW480/sh-SNHG14 and HT-29/shSNHG14 cells (Fig. 7B-C). In addition, flow cytometry

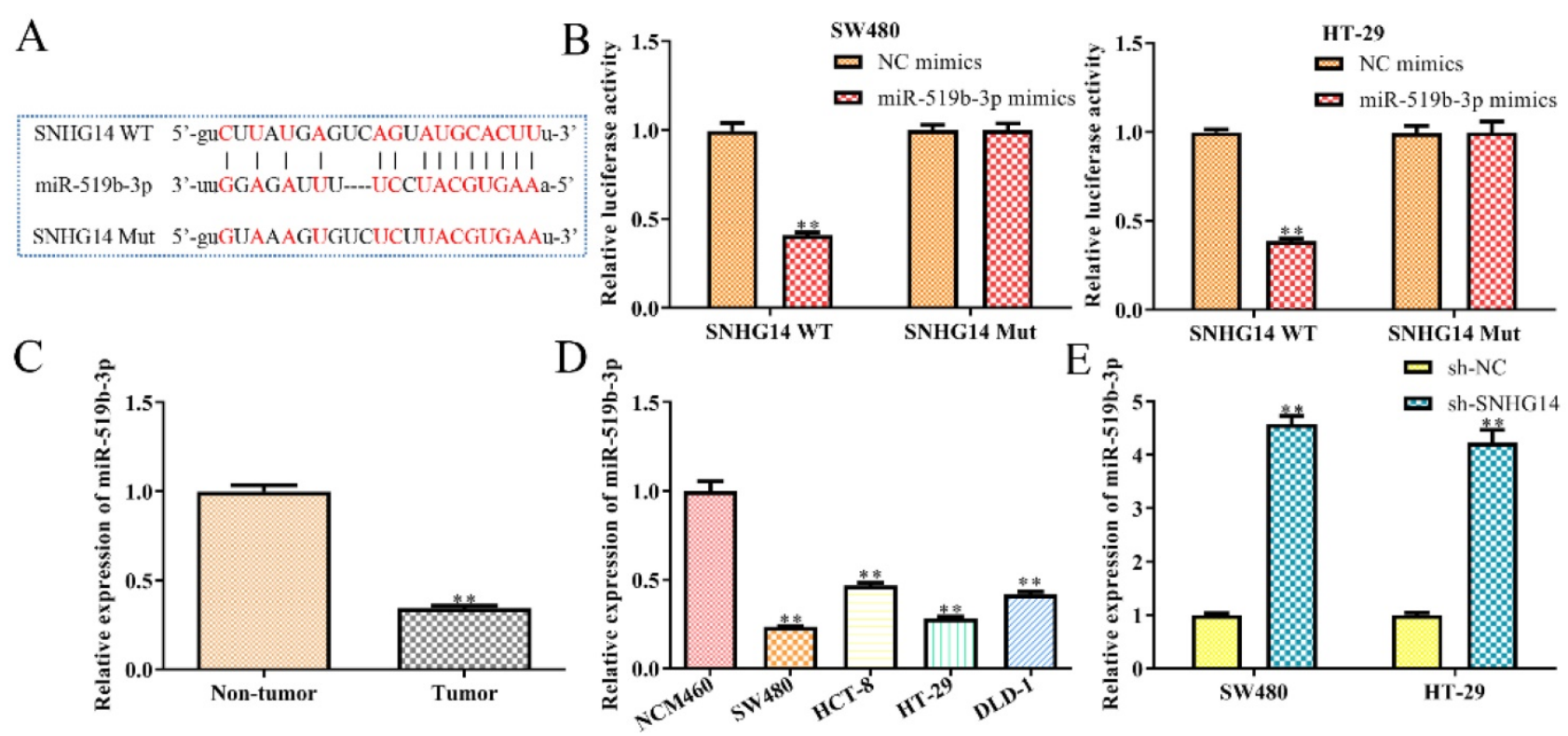

Figure 4. SNHG14 functions as a sponge for miR-519b-3p in CRC cells. (A) Bioinformatic analysis of the predicted binding site between SNHG14 and miR-519b-3p. (B) Luciferase reporter assay suggested that miR-519b-3p mimics reduced the luciferase activity of SNHG14-WT in SW480 and HT-29 cells, ***P<0.01 vs. NC mimics. (C) qRT-PCR analysis of the expression of miR-519b-3p in tumor tissues and adjacent non-tumor tissues ( $\mathrm{n}=6$ per group), $* * P<0.01$ vs. Non-tumor tissue. (D) qRT-PCR analysis of the expression of miR-519b-3p in CRC cell lines (SW480, HCT-8, HT-29 and DLD-1) and normal human colon epithelial cells (NCM460), **P<0.01 vs. NCM460. (E) qRT-PCR analysis of the expression of miR-519b-3p in SW480 and HT-29 cells transfected with sh-SNHG $14, * * p<0.01$ vs. sh-NC. Values are mean \pm SE, $n=3$ per group. 
assay showed the apoptosis of the SW480/shSNHG14 and HT-29/sh-SNHG14 cells was decreased when these cells were transfected with miR-519b-3p inhibitors, whereas sh-DDX5 reversed the reduction of apoptosis caused by miR-519b-3p inhibitors (Fig. 7D). Transwell chamber assay illustrated that
sh-DDX5 reversed miR-519b-3p inhibitors-induced activation of the migration of SW480/sh-SNHG14 and HT-29/sh-SNHG14 cells (Fig. 7E). Based on the results, we concluded that SNHG14 promotes the CRC progression by miR-519b-3p/DDX5 axis.
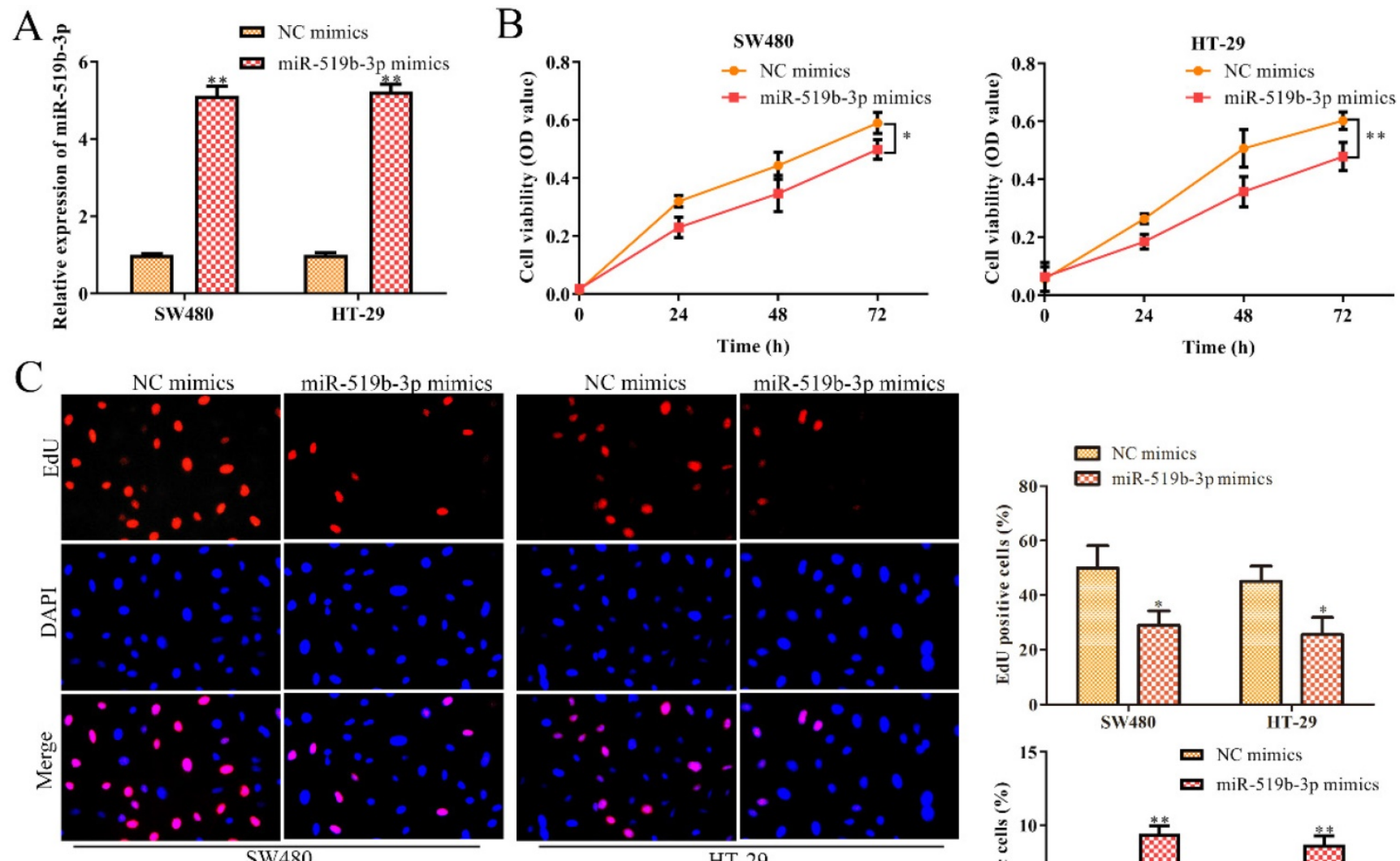

$\mathrm{D}$
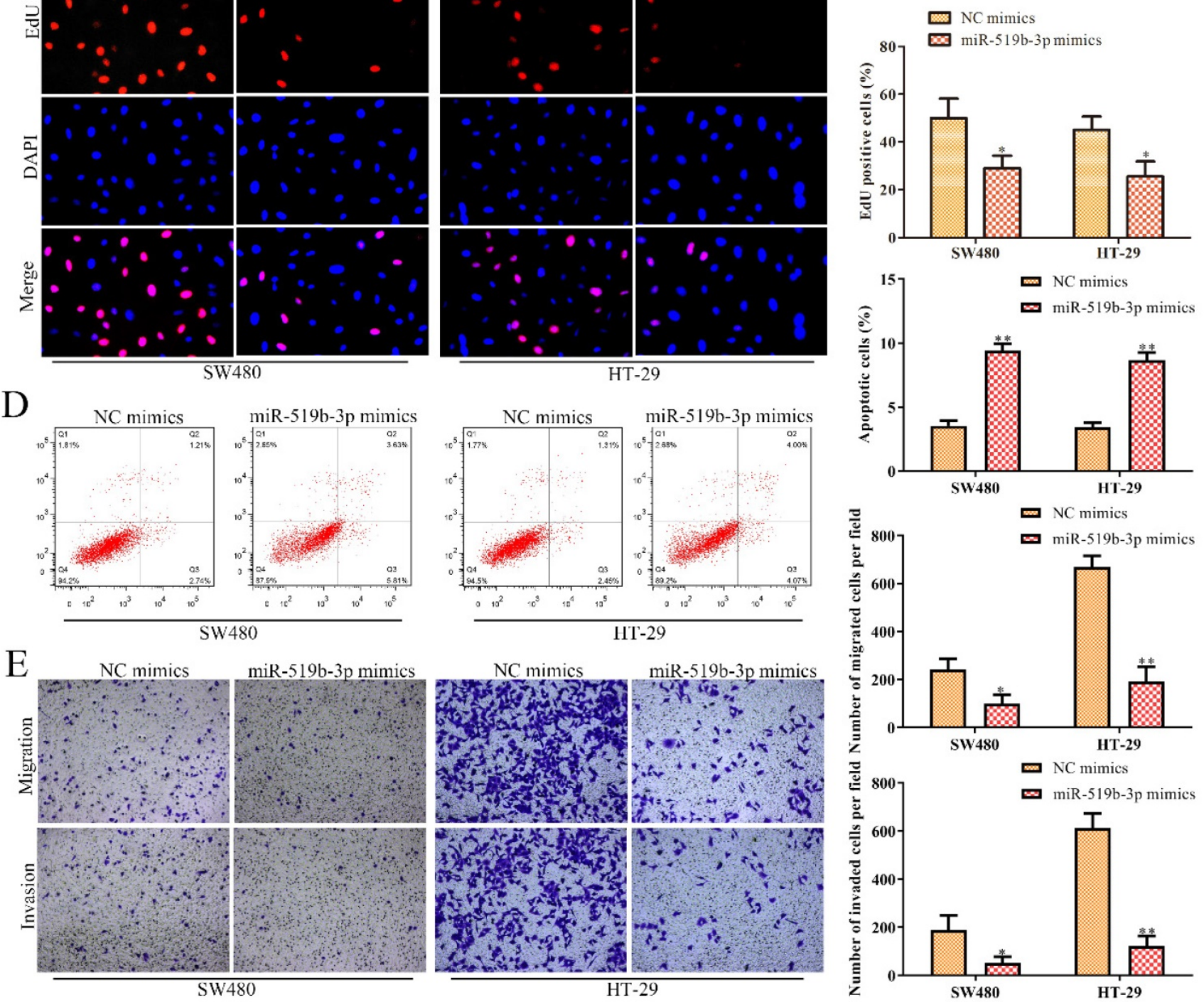

Figure 5. MiR-519b-3p inhibits cell proliferation, migration, invasion and promotes cell apoptosis in CRC. SW480 and HT-29 cells were transfected with

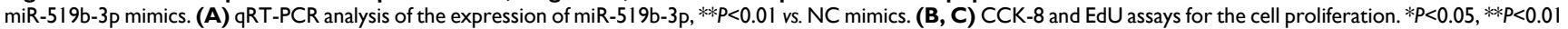
vs. NC mimics. (D) Flow cytometry analysis of the apoptosis rate. (E) Transwell chamber assay for the migration and invasion abilities, $* P<0.05$, $* * P<0.01$ vs. NC mimics. $V a$ alues are mean $\pm S E, n=3$ per group. 

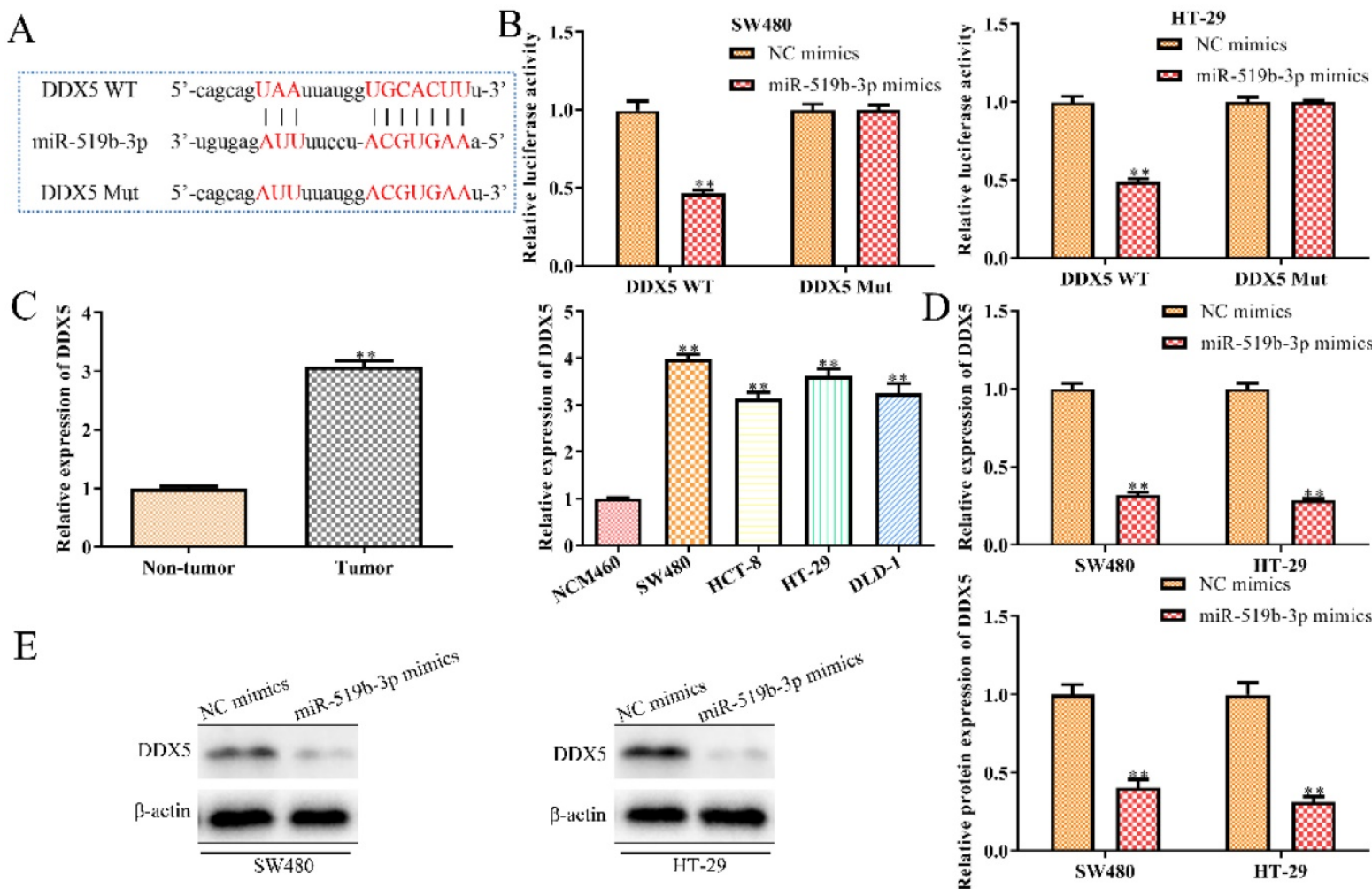

Figure 6. DDX5 is a downstream target gene of miR-519b-3p. (A) Bioinformatic analysis of the predicted binding sites of DDX5 and miR-519b-3p. (B) Luciferase reporter assay was performed at $48 \mathrm{~h}$ after transfection in SW480 and HT-29 cells with luciferase reporter plasmid containing WT or mutant form of DDX5 3'-UTR along with control or miR-519b-3p mimics. (C) qRT-PCR analysis of the expression of DDX5 in SW480 and HT-29 cells transfected with miR-519b-3p mimics. (D) Western blot analysis of the expression of DDX5 protein. **P<0.01 vs. NC mimic, $n=3$ per group. (E) qRT-PCR analysis of the expression of DDX5 in CRC tissues and cell lines. $* * P<0.01$ vs. Non-tumor tissue or NCM460 cells, $n=3$ per group. Values are mean \pm SE.

\section{Sh-SNHG 14 inhibits the CRC progression in vivo}

Given the suppressive role of sh-SNHG14 in vitro, we supposed to find out its impact on tumor growth in vivo. Human CRC cell lines were transfected by sh-SNHG14/sh-NC, the transfected HT-29 cells were injected subcutaneously into NOD-SCID mice to observe its tumorigenicity. As shown in Fig. 8A and 8B, we found that tumors from sh-SNHG14 transfected groups were obviously larger than sh-NC transfected groups. Throughout the growth of tumor, tumor lumps from the sh-SNHG14 transfected groups developed slower than those from sh-NC transfected groups (Fig. 8C). Moreover, HE staining results showed that the tumor cells are loosely from the sh-SNHG14 transfected groups compared with sh-NC transfected groups. TUNEL staining results suggested that sh-SNHG14 significantly promoted CRC cell apoptosis, and the immunohistochemistry results also showed that Ki67 protein levels in the tumor tissues generated from sh-SNHG14 transfected cells were strongly reduced (Fig. 8D). qRT-PCR results showed that sh-SNHG14 induced the decreased the expression of SNHG14, increased the expression of miR-519b-3p and decreased the expression of DDX5 (Fig. 8E).
Collectively, we concluded that sh-SNHG14 could inhibit CRC tumor growth in vivo.

\section{Discussion}

LncRNAs are crucial regulators involved in the gene expression and cancer development $[9,19,20]$. Therefore, the investigation in demonstrating the role of lncRNA could provide new insights into the identification of potential therapeutic targets in clinical applications. It is reported that lncRNA SNHG14 involved in procession of many cancers, such as pancreatic ductal adenocarcinoma, cervical cancer, renal cell carcinoma and non-small cell lung cancer, and so on [21-23]. For example, Zhang Z et al. found that SNHG14 exerts oncogenic functions in non-small cell lung cancer by acting as an miR-340 sponge [21]. However, the function and mechanism of SNHG14 in CRC remain elusive. Currently, it was reported that SNHG14 stimulated cell autophagy to facilitate cisplatin resistance of CRC by regulating miR-186/ATG14 axis [24]. In addition, SNHG14 had been reported to facilitate CRC metastasis through EZH2/EPHA7 [25]. In the current investigations, we observed that SNHG14 was highly expressed in CRC tissues as well as in the CRC cell lines, especially in SW480 and HT-29 cells. Moreover, functional assays showed that sh-SNHG14 markedly repressed cell 

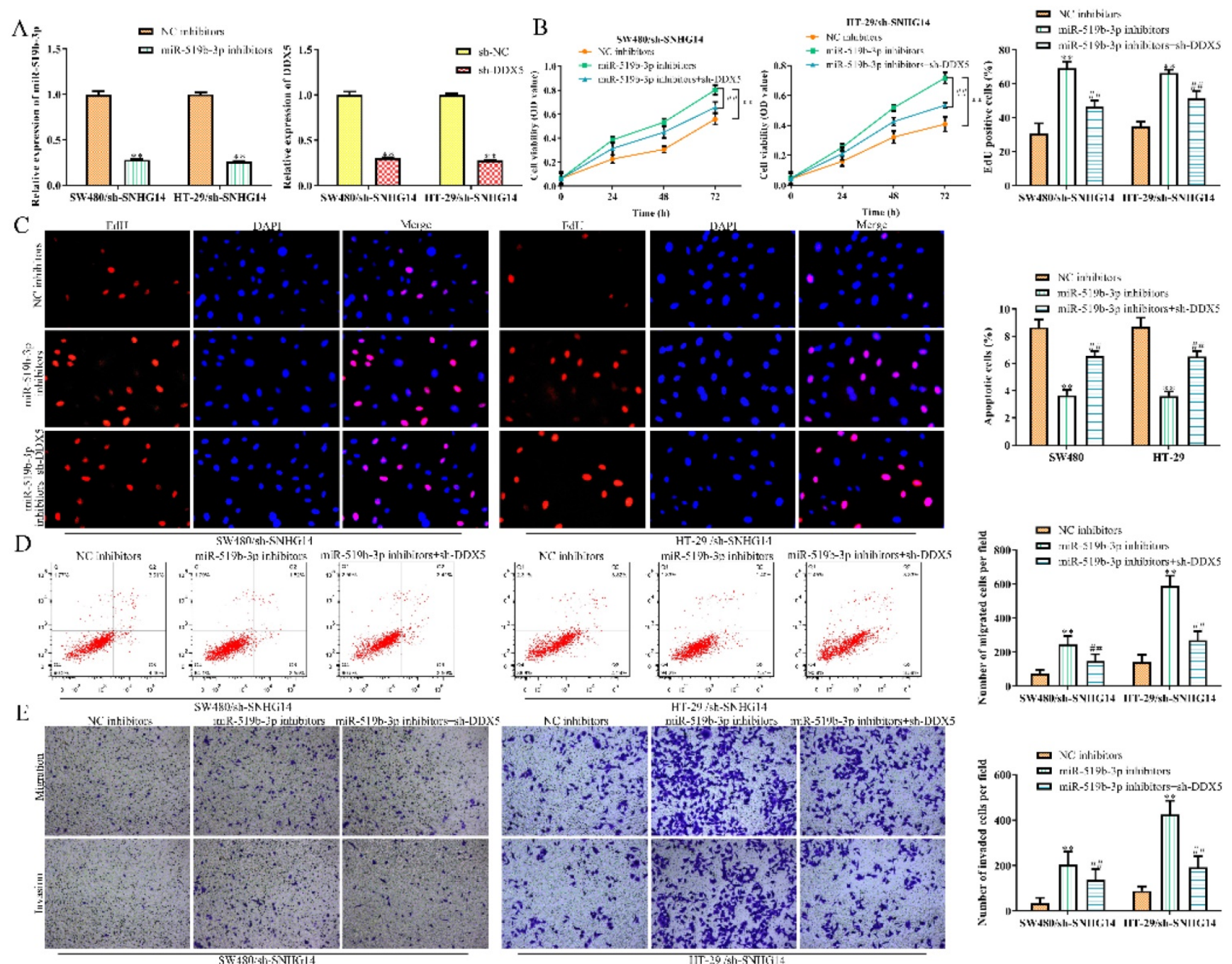

Figure 7. SNHG 14 affects CRC cell proliferation, migration, invasion and apoptosis via regulating miR-519b-3p/DDX5. (A) $q R T-P C R$ analysis of the expression of miR-519b-3p and DDX5 in SW480 and HT-29 cells transfected with miR-519b-3p inhibitors or sh-DDX5, $* * P<0.01$ vs. NC inhibitors, **P<0.01 vs. sh-NC. (B) CCK-8 assay to detect cell viability of SW480 and HT-29 cells transfected with miR-519b-3p inhibitors or/and sh-DDX5, $* * P<0.01$ vs. NC inhibitors, \# $P<0.01$ vs. miR-519b-3p inhibitors. (C) EdU assay to analyze the cell proliferation of SW480 and HT-29 cells transfected with miR-519b-3p inhibitors or/and sh-DDX5. (D) Flow cytometry analysis of the apoptosis rate of SW480 and HT-29 cells transfected with miR-519b-3p inhibitors or/and sh-DDX5, $* * P<0.01$ vs. NC inhibitors, \#\# $<<0.01$ vs. miR-519b-3p inhibitors. (E) Transwell chamber assay for the migration and invasion abilities of SW480 and HT-29 cells transfected with miR-519b-3p inhibitors or/and sh-DDX5, $* * P<0.01$ vs. NC inhibitors, \# $P<0.01$ vs. miR-519b-3p inhibitors. Values are mean $\pm \mathrm{SE}, \mathrm{n}=3$ per group.

proliferation, cell migration/invasion, and promoted cell apoptosis in CRC cell lines. All these data demonstrated that SNHG14 promoted the progression of CRC.

Accumulating evidence has shown that miRNAs act as critical regulators of cancer pathogenesis and progression [26], and lncRNAs could function as competing endogenous RNAs (ceRNAs) by sponging miRNAs to regulate the expression of specific genes targeted by miRNA, and regulate the progression of cancers. For instance, lncRNA ATB promotes the invasion-metastasis cascade in hepatocellular carcinoma [27]. LncRNA Hotair mediates tumorigenesis and metastasis in hepatocellular carcinoma through acting as an endogenous sponge for miRNA-218 and activating P14 and P16 signaling [28]. On the other hand, miR-519b-3p, a novel cancer-associated miRNA, is frequently dysregulated in a variety of diseases and plays a critical role in cellular physiology. Previous researches indicated that miR-519b-3p promotes responsiveness to preoperative chemoradiotherapy in rectal cancer patients by targeting ARID4B [29]. MiR-519b-3p inhibits the proliferation and invasion of colorectal cancer by regulating the uMtCK/Wnt signaling pathway [30]. All these studies illustrated that scientists extensively focused on the downstream mechanism of miR-519b-3p in CRC, but the upstream regulatory mechanism of miR-519b-3P is almost unknown until now. In our study, miR-519b-3p was experimentally confirmed as a target of SNHG14, and miR-519b-3p expression was down-regulated in CRC tissues and cell lines, which correlated negatively with the expression of SNHG14. Additionally, 
A

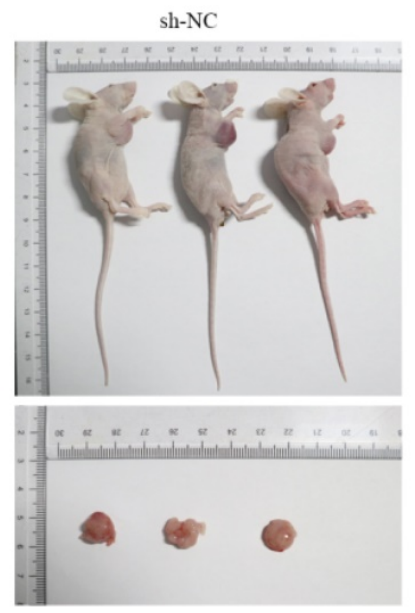

$\mathrm{D}$
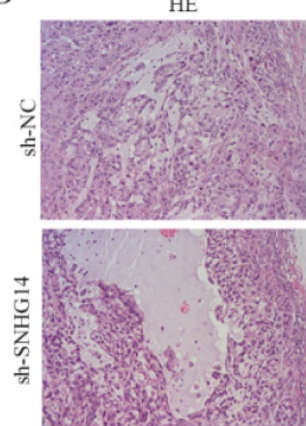
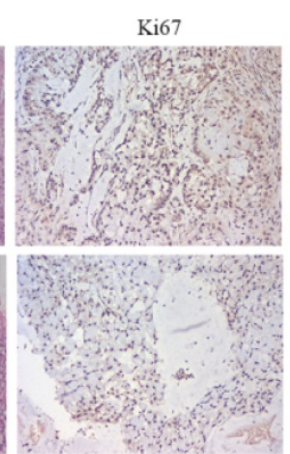
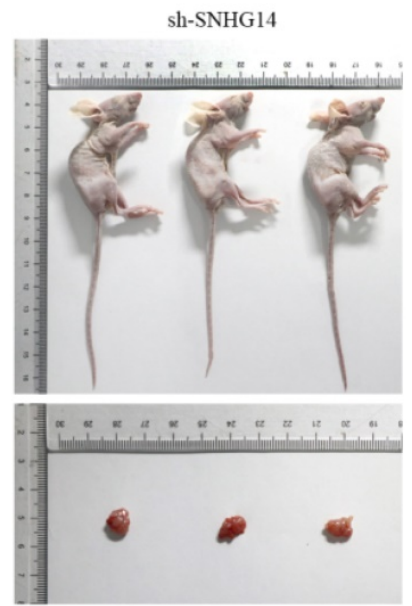

TUNEL
B
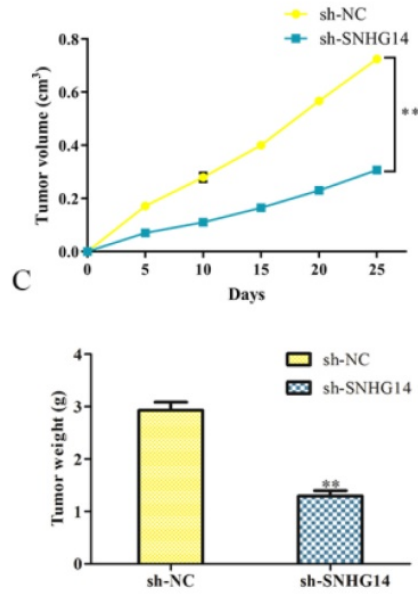

E

Figure 8. Sh-SNHG 14 inhibits the CRC progression in vivo. (A) Tumor growth of experimental NOD-SCID mice models after injected with HT-29 cells transfected with sh-SNHG 14. (B, C) Tumor volume and weights of the mice at the end of 25 days. (D) Representative Ki67 and TUNEL level in subcutaneous tumors of the mouse model. (E) Obtained tumor samples were dissociated for qRT-PCR analysis and the results exerted the SNHG14, miR-519b-3p and DDX5 expression. Values are mean \pm SE, **P<0.01 vs. sh-NC, $\mathrm{n}=6$ per group.

miR-519b-3p OE inhibits cell proliferation, migration, and invasion, as well as increased cell apoptosis rates in CRC. Similar to previous study that SNHG14 could accelerate cell proliferation, migration, invasion and suppress apoptosis in CRC cells by targeting miR-944 [31], our data suggested that SNHG14 regulated CRC progression by sponging miR-519b-3p.

In order to further explore the downstream mechanism of miR-519b-3p, we then performed bioinformatics analysis, and DDX5 was predicted as a possible target of miR-519b-3p. DDX5 exerts crucial roles in several molecular processes, including transcription, pre-mRNA processing, and miRNA processing [32]. It is reported that DDX5 plays tumor-promoter role in various cancers [33]. For instance, Du et al. [34] found that DDX5 was up-regulated in gastric cancer tissues, promoting gastric cancer progression through mTOR pathway. Ma et al. [35] observed significantly overexpressed DDX5 in human esophageal cancer cell lines while silencing of DDX5 inhibit the tumorigenesis in esophageal cancer via Wnt/ $\beta$-catenin pathway. Another research by Wang et al. [36] illustrated that DDX5 was increased in tumor tissues from lung cancer patients, playing tumor-promotive role in lung cancer. In the realm of CRC, from a cohort analysis downloaded from Starbase (http:/ / starbase.sysu.edu. $\mathrm{cn} /$ starbase2/index.php) containing 471 cancer samples and 41 normal samples, DDX5 was significantly up-regulated in CRC samples (Supplementary file). Besides, Zhang et al. [37] and Dai et al. [38] illustrated that DDX5 was highly expressed in CRC, exerting its oncogenetic role in CRC development. Consistent with previous investigation, in our work, DDX5 was experimentally confirmed as a target of miR-519b-3p, and we found DDX5 expression was significantly upregulated in CRC and negatively correlated with miR-519b-3p. Moreover, sh-DDX5 partially rescued SNHG14/ miR-519b-3p knockdown-mediated effects on cell proliferation, migration/invasion, and apoptosis in CRC cell lines. Finally, in vivo experiments further indicated that down-regulated SNHG14 greatly inhibited CRC tumor growth.

However, there were still some limitations in our study. First, from the cohort results downloaded from Starbase (http://starbase.sysu.edu.cn/starbase2/ index.php), we found that SNHG14 was decreased in CRC tumor samples compared with normal samples (Supplementary file), which was inconsistent with our 
results. In the previous studies, we also observed contradictory results concerning the expression of SNHG14 in CRC tissues. Consistent with our results, Di et al. [25], Ye et al. [39], Han et al. [24] and Pei et al. [31] verified that SNHG14 was up-regulated in CRC tumor tissues or/and cell lines, functioning as a tumor-promoter in CRC. However, Zhang et al. [40] suggested that SNHG14 was low-expressed in CRC tumor tissues and correlated with poor prognosis. Throughout various studies in cancer realm, we could conclude that SNHG14 functioned as an oncogenetic role during tumorigenesis, including endometrial cancer [41], diffuse large B cell lymphoma [42], ovarian cancer $[43,44]$, pancreatic ductal adenocarcinoma [45], cervical cancer [46,47], osteosarcoma [48], hepatocellular carcinoma [49-51], lung cancer [52,53], retinoblastoma [54] and bladder cancer [55]. In our study, we only enrolled 30 CRC patients and all the cases were collected from the Second Affiliated Hospital of Nanjing Medical University (Nanjing, China), thereby the results may bias. Hence, a larger number of CRC patients need to be collected in order to improve our results. Moreover, the clinical significance of SNHG14, miR-519b-3p and DDX5 in CRC diagnosis and prognosis should be further investigated in the future.

Taken together, these findings demonstrated that SNHG14 promotes cell proliferation and invasion in CRC through modulating miR-519b-3p/DDX5 axis. Our study facilitates the current understanding of SNHG14 function in CRC and provides a novel therapeutic target for CRC.

\section{Acknowledgements}

\section{Availability of data and materials}

The datasets used and/or analyzed during the current study are available from the corresponding author on reasonable request.

\section{Competing Interests}

The authors have declared that no competing interest exists.

\section{References}

1. Brody H. Colorectal cancer. Nature. 2015; 521: S1

2. Kornienko AE, Guenzl PM, Barlow DP, Pauler FM. Gene regulation by the act of long non-coding RNA transcription. BMC Biol. 2013; 11: 59.

3. Banfai B, Jia H, Khatun J, Wood E, Risk B, Gundling WE, Jr., et al. Long noncoding RNAs are rarely translated in two human cell lines. Genome Res. 2012; 22: 1646-57.

4. Mercer TR, Dinger ME, Sunkin SM, Mehler MF, Mattick JS. Specific expression of long noncoding RNAs in the mouse brain. Proc Natl Acad Sci U S A. 2008; 105: 716-21.

5. DiStefano JK. The Emerging Role of Long Noncoding RNAs in Human Disease. Methods Mol Biol. 2018; 1706: 91-110.

6. Sun M, Kraus WL. From discovery to function: the expanding roles of long noncoding RNAs in physiology and disease. Endocr Rev. 2015; 36: 25-64.

7. Kondo Y, Shinjo K, Katsushima K. Long non-coding RNAs as an epigenetic regulator in human cancers. Cancer Sci. 2017; 108: 1927-33.
8. Sun Z, Ou C, Liu J, Chen C, Zhou Q, Yang S, et al. YAP1-induced MALAT1 promotes epithelial-mesenchymal transition and angiogenesis by sponging miR-126-5p in colorectal cancer. Oncogene. 2019; 38: 2627-44.

9. Ma Y, Yang Y, Wang F, Moyer MP, Wei Q, Zhang P, et al. Long non-coding RNA CCAL regulates colorectal cancer progression by activating Wnt/beta-catenin signalling pathway via suppression of activator protein 2alpha. Gut. 2016; 65: 1494-504.

10. Pichler M, Rodriguez-Aguayo C, Nam SY, Dragomir MP, Bayraktar R, Anfossi $\mathrm{S}$, et al. Therapeutic potential of FLANC, a novel primate-specific long non-coding RNA in colorectal cancer. Gut. 2020; 69: 1818-31.

11. Pu J, Wei H, Tan C, Qin B, Zhang Y, Wang A, et al. Long noncoding RNA SNHG14 facilitates hepatocellular carcinoma progression through regulating miR-4673/SOCS1. Am J Transl Res. 2019; 11: 5897-904.

12. Jiao P, Hou J, Yao M, Wu J, Ren G. SNHG14 silencing suppresses the progression and promotes cisplatin sensitivity in non-small cell lung cancer. Biomed Pharmacother. 2019; 117: 109164.

13. Plosky BS. An ultraconserved lnc to miRNA processing. Mol Cell. 2014; 55: 3-4.

14. Hansen TB, Jensen TI, Clausen BH, Bramsen JB, Finsen B, Damgaard CK, et al. Natural RNA circles function as efficient microRNA sponges. Nature. 2013; 495: 384-8.

15. Abdelmohsen K, Kim MM, Srikantan S, Mercken EM, Brennan SE, Wilson GM, et al. miR-519 suppresses tumor growth by reducing HuR levels. Cell Cycle. 2010; 9: 1354-9.

16. Du C, Li DQ, Li N, Chen L, Li SS, Yang Y, et al. DDX5 promotes gastric cancer cell proliferation in vitro and in vivo through mTOR signaling pathway. Sci Rep. 2017; 7: 42876.

17. Wu N, Jiang M, Han Y, Liu H, Chu Y, Liu H, et al. O-GlcNAcylation promotes colorectal cancer progression by regulating protein stability and potential catcinogenic function of DDX5. J Cell Mol Med. 2019; 23: 1354-62.

18. Zhang $\mathrm{M}$, Weng $\mathrm{W}$, Zhang $\mathrm{Q}$, Wu $\mathrm{Y}$, Ni S, Tan C, et al. The lncRNA NEAT1 activates Wnt/beta-catenin signaling and promotes colorectal cancer progression via interacting with DDX5. J Hematol Oncol. 2018; 11: 113.

19. Taniue K, Kurimoto A, Sugimasa H, Nasu E, Takeda Y, Iwasaki K, et al. Long noncoding RNA UPAT promotes colon tumorigenesis by inhibiting degradation of UHRF1. Proc Natl Acad Sci U S A. 2016; 113: 1273-8.

20. Karapetyan AR, Buiting C, Kuiper RA, Coolen MW. Regulatory Roles for Long ncRNA and mRNA. Cancers (Basel). 2013; 5: 462-90.

21. Zhang Z, Wang $Y$, Zhang W, Li J, Liu W, Lu W. Long non-coding RNA SNHG14 exerts oncogenic functions in non-small cell lung cancer through acting as an miR-340 sponge. Biosci Rep. 2019; 39.

22. Liu G, Ye Z, Zhao X, Ji Z. SP1-induced up-regulation of lncRNA SNHG14 as a ceRNA promotes migration and invasion of clear cell renal cell carcinoma by regulating N-WASP. Am J Cancer Res. 2017; 7: 2515-25.

23. Xie F, Huang Q, Wang C, Chen S, Liu C, Lin X, et al. Downregulation of long noncoding RNA SNHG14 suppresses cell proliferation and invasion by regulating EZH2 in pancreatic ductal adenocarcinoma (PDAC). Cancer Biomark. 2020; 27: 357-64.

24. Han Y, Zhou S, Wang X, Mao E, Huang L. SNHG14 stimulates cell autophagy to facilitate cisplatin resistance of colorectal cancer by regulating miR-186/ ATG14 axis. Biomed Pharmacother. 2020; 121: 109580.

25. Di W, Weinan X, Xin L, Zhiwei $Y$, Xinyue G, Jinxue T, et al. Long noncoding RNA SNHG14 facilitates colorectal cancer metastasis through targeting EZH2-regulated EPHA7. Cell Death Dis. 2019; 10: 514.

26. Monroig Pdel C, Chen L, Zhang S, Calin GA. Small molecule compounds targeting miRNAs for cancer therapy. Adv Drug Deliv Rev. 2015; 81: 104-16.

27. Yuan JH, Yang F, Wang F, Ma JZ, Guo YJ, Tao QF, et al. A long noncoding RNA activated by TGF-beta promotes the invasion-metastasis cascade in hepatocellular carcinoma. Cancer Cell. 2014; 25: 666-81.

28. Fu WM, Zhu X, Wang WM, Lu YF, Hu BG, Wang H, et al. Hotair mediates hepatocarcinogenesis through suppressing miRNA-218 expression and activating P14 and P16 signaling. J Hepatol. 2015; 63: 886-95.

29. Luo J, Liu L, Zhou N, Shen J, Sun Q, Zhu Y, et al. miR-519b-3p promotes responsiveness to preoperative chemoradiotherapy in rectal cancer patients by targeting ARID4B. Gene. 2018; 655: 84-90.

30. Zhang Y, Sun M, Chen Y, Li B. MiR-519b-3p Inhibits the Proliferation and Invasion in Colorectal Cancer via Modulating the uMtCK/Wnt Signaling Pathway. Front Pharmacol. 2019; 10: 741

31. Pei Q, Liu GS, Li HP, Zhang Y, Xu XC, Gao H, et al. Long noncoding RNA SNHG14 accelerates cell proliferation, migration, invasion and suppresses apoptosis in colorectal cancer cells by targeting miR-944/KRAS axis through PI3K/AKT pathway. Eur Rev Med Pharmacol Sci. 2019; 23: 9871-81.

32. Bourgeois $\mathrm{CF}$, Mortreux F, Auboeuf D. The multiple functions of RNA helicases as drivers and regulators of gene expression. Nat Rev Mol Cell Biol. 2016; 17: 426-38

33. Nyamao RM, Wu J, Yu L, Xiao X, Zhang FM. Roles of DDX5 in the tumorigenesis, proliferation, differentiation, metastasis and pathway regulation of human malignancies. Biochim Biophys Acta Rev Cancer. 2019; 1871: 85-98.

34. Du C, Li DQW, Li N, Chen L, Li SS, Yang Y, et al. DDX5 promotes gastric cancer cell proliferation in vitro and in vivo through mTOR signaling pathway. Sci Rep. 2017; 7: 42876.

35. Wu D, Xue WN, Li X, Yu ZW, Gu XY, Tong JX, et al. Long noncoding RNA SNHG14 facilitates colorectal cancer metastasis through targeting EZH2-regulated EPHA7. Cell Death Dis. 2019; 10: 514. 
36. Wang ZD, Luo ZH, Zhou L, Li XF, Jiang T, Fu EQ. DDX5 promotes proliferation and tumorigenesis of non-small-cell lung cancer cells by activating $\beta$-catenin signaling pathway. Cancer Sci. 2015; 106: 1303-1312.

37. Zhang $\mathrm{M}$, Weng WW, Zhang QY, Wu Y, Ni SJ, Tan $\mathrm{C}$, et al. The lncRNA NEAT1 activates $W n t / \beta$-catenin signaling and promotes colorectal cancer progression via interacting with DDX5. J Hematol Oncol. 2018; 11: 113.

38. Dai L, Pan GD, Liu XJ, Huang J, Jiang ZQ, Zhu XB, et al. High expression of ALDOA and DDX5 are associated with poor prognosis in human colorectal cancer. Cancer Manag Res. 2018; 10: 1799-1806.

39. Ye T, Zhang N, Wu WY, Yang B, Wang JH, Huang WQ, et al. SNHG14 promotes the tumorigenesis and metastasis of colorectal cancer through miR-32-5p/SKIL axis. In vitro Cell Dev Biol Anim. 2019; 55: 812-820.

40. Zhang W, Duan WF, Mo ZF, Wang JN, Yang WB, Wu WR, et al. Upregulation of SNHG14 suppresses cell proliferation and metastasis of colorectal cancer by targeting miR-92b-3p. J Cell Biochem. 2020; 121(2): 1998-2008.

41. Wang GF, Wen LN. LncRNA SNHG14 promotes proliferation of endometrial cancer through regulating microRNA-655-3p. Eur Rev Med Pharmacol Sci. 2020; 24: 10410-10418.

42. Zhao LN, Liu Y, Zhang JB, Liu Y, Qi Q. LncRNA SNHG14/miR-5590-3p/ZEB1 positive feedback loop promoted diffuse large $B$ cell lymphoma progression and immune evasion through regulating PD-1/PD-L1 checkpoint. Cell Death Dis. 2019; 10: 731.

43. Zhao YL, Huang YM. LncSNHG14 promotes ovarian cancer by targeting microRNA-125a-5p. Eur Rev Med Pharmacol Sci. 2019; 23: 3235-3242.

44. Zhao JL, Wang CL, Liu YL, Zhang GY. Long noncoding RNA SNHG14 enhances migration and invasion of ovarian cancer by upregulating DGCR8. Eur Rev Med Pharmacol Sci. 2019; 23: 10226-10233.

45. Xie F, Huang Q, Wang C, Chen SW, Liu CH, Lin XS, et al. Downregulation of long noncoding RNA SNHG14 suppresses cell proliferation and invasion by regulating EZH2 in pancreatic ductal adenocarcinoma (PDAC). Cancer Biomark. 2020; 27: 357-364

46. Ji NN, Wang YH, Bao GL, Yan JL, Ji S. LncRNA SNHG14 promotes the progression of cervical cancer by regulating miR-206/YWHAZ. Pathol Res Pract. 2019; 215: 668-675.

47. Zhang $\mathrm{YY}, \mathrm{Li} \mathrm{M}, \mathrm{Xu} \mathrm{YD}$, Shang J. LncRNA SNHG14 promotes the development of cervical cancer and predicts poor prognosis. Eur Rev Med Pharmacol Sci. 2019; 23(9): 3664-3671.

48. Hou XK, Mao JS. Long noncoding RNA SNHG14 promotes osteosarcoma progression via miR-433-3p/FBXO22 axis. Biochem Biophys Res Commun. 2020; 523: 766-772.

49. Zhang $\mathrm{H}, \mathrm{Xu} \mathrm{HB}$, Kurban E, Luo HW. LncRNA SNHG14 promotes hepatocellular carcinoma progression via H3K27 acetylation activated PABPC1 by PTEN signaling. Cell Death Dis. 2020; 11: 646.

50. Xu XY, Song FH, Jiang XW, Hong $\mathrm{H}$, Fei Q, Jin ZK, et al. Long non-coding RNA SNHG14 contributes to the development of hepatocellular carcinoma via sponging miR-217. Onco Targets Ther. 2020; 13: 4865-4876.

51. Lin RX, Zhan GF, Wu JC, Fang H, Yang SL. LncRNA SNHG14 Sponges miR-206 to Affect Proliferation, Apoptosis, and Metastasis of Hepatocellular Carcinoma Cells by Regulating SOX9. Dig Dis Sci. 2021; 29.

52. Zhao L, Zhang XD, Shi YT, Teng TL. LncRNA SNHG14 contributes to the progression of NSCLC through miR-206/G6PD pathway. Thorac Cancer. 2020; 11: 1202-1210.

53. Zhang ZH, Wang Y, Zhang W, Li JY, Liu WL, Lu WM. Long non-coding RNA SNHG14 exerts oncogenic functions in non-small cell lung cancer through acting as an miR-340 sponge. Biosci Rep. 2019; 39: BSR20180941.

54. Sun XW, Shen H, Liu SB, Gao J, Zhang SY. Long noncoding RNA SNHG14 promotes the aggressiveness of retinoblastoma by sponging microRNA-124 and thereby upregulating STAT3. Int J Mol Med. 2020; 45: 1685-1696.

55. Li J, Wang AS, Wang S, Wang CY, Xue S, Guan H, et al. LncSNHG14 promotes the development and progression of bladder cancer by targeting miRNA-150-5p. Eur Rev Med Pharmacol Sci. 2019; 23: 1022-1029. 